\title{
Estimating the Risk of Severe Peanut Allergy Using Clinical Background and IgE Sensitization Profiles
}

\section{OPEN ACCESS}

Edited by:

Kari Christine Nadeau,

Stanford University, United States

Reviewed by:

Komei Ito,

Aichi Child Health and Medical

General Center, Japan

Andrew John Long,

Stanford University, United States

*Correspondence:

Sarah A. Lyons

s.a.lyons@umcutrecht.n!

${ }^{\dagger}$ These authors share first authorship

Specialty section:

This article was submitted to

Food Allergy,

a section of the journal

Frontiers in Allergy

Received: 22 February 2021 Accepted: 06 May 2021

Published: 07 June 2021

Citation:

Datema MR, Lyons SA,

Fernández-Rivas M, Ballmer-Weber B,

Knulst AC, Asero R, Barreales $L$,

Belohlavkova S, de Blay F, Clausen M,

Dubakiene R, Fernández-Perez $C$

Fritsche $P$, Gislason D,

Hoffmann-Sommergruber $K$, Jedrzejczak-Czechowicz M,

Jongejan L, Kowalski ML,

Kralimarkova TZ, Lidholm J,

Papadopoulos NG, Popov TA,

Prado Nd, Purohit A, Reig I,

Seneviratne SL, Sinaniotis A,

Vassilopoulou E, Versteeg $S A$,

Vieths S, Welsing PMJ, Mills ENC,

Le T-M, Zwinderman AH and van

Ree $R$ (2021) Estimating the Risk of

Severe Peanut Allergy Using Clinical

Background and IgE Sensitization

Profiles. Front. Allergy 2:670789.

doi: 10.3389/falgy.2021.670789
Mareen R. Datema ${ }^{1,2 \dagger}$, Sarah A. Lyons ${ }^{3 * \dagger}$, Montserrat Fernández-Rivas ${ }^{4}$,

Barbara Ballmer-Weber ${ }^{5,6,7}$, André C. Knulst ${ }^{3}$, Riccardo Asero ${ }^{8}$, Laura Barreales ${ }^{9}$, Simona Belohlavkova ${ }^{10}$, Frédéric de Blay ${ }^{11}$, Michael Clausen ${ }^{12}$, Ruta Dubakiene ${ }^{13}$, Cristina Fernández-Perez ${ }^{9}$, Philipp Fritsche ${ }^{5}$, David Gislason ${ }^{12}$,

Karin Hoffmann-Sommergruber ${ }^{14}$, Monika Jedrzejczak-Czechowicz ${ }^{15}$, Laurian Jongejan ${ }^{1}$, Marek L. Kowalski ${ }^{15}$, Tanya Z. Kralimarkova ${ }^{16}$, Jonas Lidholm ${ }^{17}$,

Nikolaos G. Papadopoulos 18,19, Todor A. Popov ${ }^{20}$, Nayade del Prado ${ }^{9}$, Ashok Purohit ${ }^{11}$, Isabel Reig ${ }^{4}$, Suranjith L. Seneviratne ${ }^{21}$, Athanassios Sinaniotis ${ }^{18}$, Emilia Vassilopoulou ${ }^{22}$, Serge A. Versteeg ${ }^{1}$, Stefan Vieths ${ }^{23}$, Paco M. J. Welsing ${ }^{24}$, E. N. Clare Mills ${ }^{25}$, Thuy-My Le ${ }^{3}$, Aeilko H. Zwinderman ${ }^{2}$ and Ronald van Ree ${ }^{1,26}$

${ }^{1}$ Department of Experimental Immunology, Amsterdam University Medical Center, Amsterdam, Netherlands, ${ }^{2}$ Department of Clinical Epidemiology, Biostatistics, and Bioinformatics, Amsterdam University Medical Center, Amsterdam, Netherlands, ${ }^{3}$ Department of Dermatology and Allergology, University Medical Center Utrecht, Utrecht University, Utrecht, Netherlands, ${ }^{4}$ Allergy Department, Hospital Clinico San Carlos, Instituto de Investigacion Sanitario San Carlos, Madrid, Spain, ${ }^{5}$ Allergy Unit, Department of Dermatology, University Hospital, Zurich, Switzerland, ${ }^{6}$ Faculty of Medicine, University of Zurich, Zurich, Switzerland, ${ }^{7}$ Clinic for Dermatology and Allergology, Kantonsspital St. Gallen, St. Gallen, Switzerland, ${ }^{8}$ Ambulatorio di Allergologia, Clinica San Carlo, Paderno Dugnano, Italy, ${ }^{9}$ Clinical Epidemiology Unit, Preventive Medicine Department, Hospital Clinico San Carlos, Instituto de Investigacion Sanitario San Carlos, Madrid, Spain, ${ }^{10}$ Department of Allergology and Immunology, Faculty of Medicine in Pilsen, Charles University, Prague, Czechia, ${ }^{11}$ Allergy Division, Chest Disease Department, Strasbourg University Hospital, Strasbourg, France, ${ }^{12}$ Faculty of Medicine, Landspitali University Hospital, University of Iceland, Reykjavik, Iceland, ${ }^{13}$ Medical Faculty, Vilnius University, Vilnius, Lithuania, ${ }^{14}$ Department of Pathophysiology and Allergy Research, Medical University of Vienna, Vienna, Austria, ${ }^{15}$ Department of Immunology, Rheumatology and Allergy, Faculty of Medicine, Medical University of Lodz, Lodz, Poland, ${ }^{16}$ Clinical Center of Allergology, Medical University of Sofia, Sofia, Bulgaria, ${ }^{17}$ Thermo Fisher Scientific, Uppsala, Sweden, ${ }^{18}$ Allergy Department, $2 n d$ Pediatric Clinic, University of Athens, Athens, Greece, ${ }^{19}$ Division of Infection, Immunity \& Respiratory Medicine, University of Manchester, Manchester, United Kingdom, ${ }^{20}$ Clinic of Occupational Diseases, University Hospital Sv. Ivan Rilski, Sofia, Bulgaria, ${ }^{21}$ Institute of Immunity and Transplantation, University College London, London, United Kingdom, ${ }^{22}$ Department of Nutritional Sciences and Dietetics, International Hellenic University, Thessaloniki, Greece, ${ }^{23}$ Paul-Ehrlich-Institut, Federal Institute for Vaccines and Biomedicines, Langen, Germany, ${ }^{24}$ Division of Internal Medicine and Dermatology, University Medical Center Utrecht, Utrecht University, Utrecht, Netherlands, ${ }^{25}$ Division of Infection, Immunity and Respiratory Medicine, Manchester Institute of Biotechnology, University of Manchester, Manchester, United Kingdom, ${ }^{26}$ Department of Otorhinolaryngology, Amsterdam University Medical Center, Amsterdam, Netherlands

Background: It is not well-understood why symptom severity varies between patients with peanut allergy (PA).

Objective: To gain insight into the clinical profile of subjects with mild-to-moderate and severe PA, and investigate individual and collective predictive accuracy of clinical background and lgE to peanut extract and components for PA severity.

Methods: Data on demographics, patient history and sensitization at extract and component level of 393 patients with probable PA (symptoms $\leq 2 \mathrm{~h}+\lg$ E sensitization) from 12 EuroPrevall centers were analyzed. Univariable and penalized multivariable regression analyses were used to evaluate risk factors and biomarkers for severity.

Results: Female sex, age at onset of PA, symptoms elicited by skin contact with peanut, family atopy, atopic dermatitis, house dust mite and latex allergy were independently associated with severe PA; birch pollen allergy with mild-to-moderate PA. 
The cross-validated AUC of all clinical background determinants combined (0.74) was significantly larger than the AUC of tests for sensitization to extract (0.63) or peanut components (0.54-0.64). Although larger skin prick test wheal size, and higher lgE to peanut extract, Ara h 1 and Ara h 2/6, were associated with severe PA, and higher lgE to Ara h 8 with mild-to-moderate PA, addition of these measurements of sensitization to the clinical background model did not significantly improve the AUC.

Conclusions: Models combining clinical characteristics and lgE sensitization patterns can help establish the risk of severe reactions for peanut allergic patients, but clinical background determinants are most valuable for predicting severity of probable PA in an individual patient.

Keywords: EuroPrevall, iFAAM, peanut allergy, severity, prediction, clinical background, IgE, component-resolved diagnostics

\section{INTRODUCTION}

Patients with peanut allergy (PA) often require strict elimination diets to prevent potentially severe allergic reactions. Beyond levels of exposure, it is not well-understood why symptom severity varies between patients (1).

To gain insight into severity of PA in a particular patient, accurate clinical evaluation is essential. Besides patient history, routine diagnostic tests include extract-based skin prick testing (SPT) and serum $\operatorname{IgE}$ measurements. There is conflicting evidence on the usefulness of SPT and IgE levels for predicting severity of PA (2-5). In recent years, serum IgE testing using whole food extracts has been complemented with allergen component testing. For peanut, IgE to Ara $\mathrm{h} 2$ has been demonstrated to better distinguish PA from tolerance than $\operatorname{IgE}$ to peanut extract (6-14). Some studies have reported a relationship between IgE levels to Ara h 2 and severity of PA $(7,11,14-16)$, whereas other studies report no clear difference $(6,12,17,18)$. Food challenge, preferably double-blind placebo-controlled food challenge (DBPCFC), is the reference standard for confirming presence and severity of PA. However, due to the burdensome and resource-intensive nature of food challenge, daily practice diagnosis is often based on a suggestive patient history in combination with IgE sensitization (i.e., probable PA) (19).

Peanut and tree nuts are reportedly the most common causes of food-induced anaphylaxis (1). In recent papers on hazelnut allergy (20) and walnut allergy (21), we set out to develop prediction models in which a patient's demographic and clinical background is combined with results from routine extractbased tests and from component-resolved diagnostics (CRD). For both tree nuts, models combining clinical background information with measures of IgE-sensitization were shown to improve the accuracy of predicting severe reactions significantly compared with clinical variables, IgE to extract, or IgE to allergen components alone. Although several previous studies have evaluated the predictive accuracy of combined clinical and

Abbreviations: SPT, Skin prick test; AD, Atopic Dermatitis; CRD, Componentresolved diagnosis; DBPCFC, Double-blind placebo-controlled food challenge; LTP, Lipid transfer protein; OAS, Oral allergy syndrome; OR, Odds Ratio. serological information for predicting PA $(6,7,22,23)$, the focus is rarely on prediction of severity. Petterson et al. developed a model for severe PA based on clinical characteristics and serum IgE against peanut extract, but did not assess contribution of CRD, and included only children (22).

In the present study, we evaluated data collected from predominantly adult patients reporting PA during the EuroPrevall outpatient clinic surveys in 12 different European cities (16), using an approach comparable to that in previous evaluations for hazelnut and walnut. In a subset of these patients that underwent DBPCFC, Ballmer-Weber and colleagues previously reported that systemic reactions occurred significantly more frequently in subjects sensitized to peanut extract ( $\operatorname{IgE}$ $\geq 0.35 \mathrm{kU} / \mathrm{L})$ or to Ara h 2 (IgE $\geq 1.0 \mathrm{kU} / \mathrm{L})$ (16). Our aim was to further investigate the association of demographics, clinical background, and markers of peanut sensitization, with the severity of PA, and to subsequently develop prediction models using all this information to improve discriminatory ability for estimating the risk of severe reactions.

\section{METHODS}

\section{Study Design and Population}

Twelve European allergy centers in Athens (Greece), Łódz (Poland), Madrid (Spain), Manchester (United Kingdom), Milan (Italy), Prague (Czech Republic), Reykjavik (Iceland), Sofia (Bulgaria), Strasbourg (France), Utrecht (The Netherlands), Vilnius (Lithuania) and Zürich (Switzerland), enrolled patients with a history of food allergy (FA) in the EuroPrevall outpatient clinic study. Each local ethical committee approved the study. Recruitment took place between 2006 and 2009. Informed consent was documented for all patients before enrollment in the study. For the current study, we included all patients reporting adverse reactions within $2 \mathrm{~h}$ of ingestion of peanut.

\section{Clinical Evaluation}

The methodology of the EuroPrevall outpatients study has been described in detail elsewhere (24). All patients underwent an extensive questionnaire, which focused on reaction characteristics and allergic comorbidities, and was 
administered and interpreted by trained physicians. Skin prick test (SPT) reactivity to peanut extract was assessed using a commercially available extract (ALK-Abelló, Madrid, Spain). Serum samples were collected locally in each center, and analyzed by ImmunoCAP (Thermo Fisher Scientific, Uppsala, Sweden) at the Paul-Ehrlich Institute (Langen, Germany). All available sera were tested for sensitization to peanut extract, as well as to other food and inhalant allergens (24). A custom-made microarray chip, technically resembling the ImmunoCAP ISAC test, was used to test for sensitization to food allergen components, amongst which were peanut allergens nAra h 1 (7S globulin), nAra h 2/6 (2S albumin), nAra h 3 (11S globulin), and rAra h 8 (pathogenesis-related protein family 10 [PR-10] protein) $(24,25)$. DBPCFC was carried out in all consenting subjects by trained clinicians as described previously (26).

\section{Definitions}

Patients who, along with symptoms within $2 \mathrm{~h}$ of peanut ingestion, had IgE sensitization to peanut, as measured by positive SPT, ImmunoCAP or microarray, were defined as having probable PA. SPT allergen/histamine wheal ratios were considered positive at a ratio $\geq 0.5$, IgE in ImmunoCAP at levels $\geq 0.35 \mathrm{kU}_{\mathrm{A}} / \mathrm{L}$, and IgE in microarray at levels $\geq 0.3 \mathrm{ISU} / \mathrm{L}$.

Severity of symptoms, as determined from the physicianadministered questionnaire, was classified into two groups: mildto-moderate if isolated oral allergy symptoms or symptoms of the skin, eyes, upper airway and/or gastrointestinal system occurred; severe in case of symptoms of the lower airway (either laryngeal or bronchial), cardiovascular or neurological system $(27,28)$. Skin symptoms included urticaria, angioedema, erythema/flushing, or itching; eye symptoms pertained to conjunctivitis; upper airway symptoms pertained to rhinitis; and gastrointestinal symptoms included dysphagia, stomach pain, cramps, nausea, vomiting, or diarrhea. Lower airway symptoms consisted of throat tightness, dysphonia, dyspnoea, wheezing, cough, or chest tightness; cardiovascular symptoms included cardiac arrhythmia, myocardial ischaemia, or hypotension; neurological symptoms comprised disorientation/ confusion, dizziness, seizures, incontinence, or loss of consciousness. Severity was based on each participant's most severe reaction to peanut.

Patients with proven sensitization in SPT or ImmunoCAP matching their reported rhinoconjunctivitis or asthma symptoms to birch, grass, mugwort, house dust mite (HDM) or latex were considered to be allergic to the respective allergen sources.

\section{Statistical Analysis}

All analyses were performed in subjects with probable PA. In univariable analysis, differences in demographic factors and clinical background (age, sex, age at onset of PA $[<14$ $v s$. $\geq 14$ years], symptoms upon skin contact with peanuts [did skin contact with peanut induce adverse reactions, e.g., contact urticaria, dermatitis, rhinoconjunctivitis, bronchospasm or anaphylaxis?], first degree family members with atopy, $A D$ [ever], allergy to pollen, HDM or latex, and sensitization to cats or dogs), results from extract-based testing (SPT and ImmunoCAP with peanut extract), and results from CRD (microarray Ara $\mathrm{h} 1$, $2 / 6,3$, and 8 ), were evaluated using chi-square tests, independent sample $t$-tests, or Mann-Whitney $U$-tests where appropriate. Bonferroni corrections were used to correct for multiple testing.

Multivariable analyses were performed to identify the most relevant set of predictors for severity of probable PA. To limit overfitting and improve generalizability, the Least Absolute Shrinkage and Selection Operator (Lasso) regression approach was chosen. This method selects only the most discriminative combination of variables, and applies cross-validation to shrink regression coefficients (29). To ensure use of all data, missing data were imputed ten-fold using the mice package in $\mathrm{R}$ software. Details on missing data and included covariates are available from Supplementary Table 1. Lasso regression was repeated on each of the 10 datasets. Predictor variables selected in at least 7/10 imputed datasets were included. Bootstrapping was used to estimate $95 \%$ confidence intervals (CI) for each coefficient. Results were pooled using Rubin's rules.

A stepwise approach to model building was taken, and the Lasso selection process was applied in each step. In model I, all variables on demographics and clinical background were entered. In model II, peanut extract-based test results (SPT [wheal ratios] and ImmunoCAP [IgE levels]) were added to the selected model I variables. In model III, peanut CRD results were entered, along with the variables selected in model II. Finally, to explore if knowledge of IgE levels to plant source food extracts and components other than peanut could improve prediction of PA severity, ImmunoCAP and CRD results related to sensitization to soybean, lentil, hazelnut, walnut, sesame seed, peach, apple, kiwi, tomato, carrot, and celery, were entered in a final step, after fixing the variables selected in model III. The discriminatory ability of the resulting regression models to distinguish between mild-to-moderate and severe probable PA was quantified by area under the receiving operating curve (AUC) estimators. AUCs were compared using DeLong's test (30).

For comparative purposes, Lasso regression analyses were repeated in a subgroup consisting of only subjects with clinically determined symptom severity based on DBPCFC and subjects excluded from DBPCFC because of a convincing history of severe life-threatening anaphylaxis. The latter subjects were defined as having had a reaction involving hypotension, severe bronchospasm or laryngeal edema within $2 \mathrm{~h}$ of peanut ingestion, leading to emergency treatment (24). The principal investigators in Madrid, Utrecht and Zurich reviewed these severe reactions and all agreed upon exclusion of these subjects from DBPCFC, making these patients' history particularly reliable. Subjects with a negative DBPCFC outcome and placebo-reactors were grouped with the mild-to-moderate DBPCFC reactors for this subgroup analysis.

Analyses were conducted with $\mathrm{R}$ version 3.4.1.

\section{RESULTS}

Of the 517 subjects reporting symptoms within $2 \mathrm{~h}$ of ingestion of peanut, $393(76 \%)$ had probable PA. Overall, $216(55 \%)$ had mild-to-moderate and 177 (45\%) had severe probable PA (Table 1, Supplementary Figure 1). Of the subjects with mildto-moderate probable PA, 89/216 (41\%) had isolated oral allergy symptoms (OAS). 
TABLE 1 | Characteristics of subjects with probable PA.

\begin{tabular}{|c|c|c|c|}
\hline Variable & $\begin{array}{l}\text { Mild-to-moderate } \\
\qquad(N=216)\end{array}$ & $\begin{array}{l}\text { Severe } \\
(N=177)\end{array}$ & $p$ \\
\hline \multicolumn{4}{|l|}{ Demographics } \\
\hline $\begin{array}{l}\text { Age at visit in years, mean } \\
( \pm S D)\end{array}$ & $28.2( \pm 14.3)$ & $24.8( \pm 13.7)$ & 0.019 \\
\hline Age $<14$ years & $30 / 216$ (13.9) & 39/177 (22.0) & 0.048 \\
\hline Female sex & $126 / 216(58.3)$ & 106/177 (59.9) & 0.835 \\
\hline \multicolumn{4}{|l|}{ Clinical background } \\
\hline $\begin{array}{l}\text { Age at onset of symptoms } \\
<14 \text { years }\end{array}$ & $86 / 211(40.8)$ & 113/174 (64.9) & $<0.001^{*}$ \\
\hline $\begin{array}{l}\text { Symptoms upon skin } \\
\text { contact with peanut }\end{array}$ & 10/192 (5.2) & 48/146 (32.9) & $<0.001^{*}$ \\
\hline $\begin{array}{l}\text { Family history of atopic } \\
\text { disease }\end{array}$ & $131 / 210(62.4)$ & 123/176 (69.9) & 0.150 \\
\hline Atopic dermatitis & 62/212 (29.2) & 89/175 (50.9) & $<0.001^{*}$ \\
\hline Birch pollen allergy ${ }^{\ddagger}$ & $124 / 213(58.2)$ & $81 / 172(47.1)$ & 0.038 \\
\hline Grass pollen allergy ${ }^{\ddagger}$ & $124 / 213(58.2)$ & 109/172 (63.4) & 0.355 \\
\hline Mugwort pollen allergy ${ }^{\ddagger}$ & $42 / 213(19.7)$ & 23/172 (13.4) & 0.130 \\
\hline House dust mite allergy ${ }^{\ddagger}$ & $98 / 201(48.8)$ & 106/160 (66.2) & 0.001 \\
\hline Latex allergy $y^{\ddagger}$ & 10/195 (5.1) & 23/165 (13.9) & 0.007 \\
\hline Cat/dog sensitization ${ }^{\ddagger}$ & $146 / 215(67.9)$ & 137/175 (78.3) & 0.030 \\
\hline \multicolumn{4}{|l|}{ Peanut sensitization ${ }^{\S}$} \\
\hline \multicolumn{4}{|l|}{ SPT peanut extract } \\
\hline Positive & 176/212 (83.0) & 144/175 (82.3) & 0.956 \\
\hline $\begin{array}{l}\text { Allergen/histamine wheal } \\
\text { ratio, median (IQR) }\end{array}$ & $0.78(0.57-1.00)$ & $1.07(0.64-1.80)$ & $<0.001^{*}$ \\
\hline \multicolumn{4}{|c|}{ ImmunoCAP peanut extract } \\
\hline Positive & 144/209 (68.9) & 140/167 (83.8) & $0.001^{*}$ \\
\hline IgE level, median (IQR) & $0.95(0.22-3.23)$ & $2.21(0.75-12.84)$ & $<0.001^{*}$ \\
\hline \multicolumn{4}{|c|}{ Microarray peanut allergens ${ }^{* *}$} \\
\hline \multicolumn{4}{|l|}{ Ara h 1} \\
\hline Positive & 26/176 (14.8) & $54 / 144(37.5)$ & $<0.001^{*}$ \\
\hline IgE level, median (IQR) & $0.00(0.00-0.00)$ & $0.00(0.00-0.83)$ & 0.004 \\
\hline \multicolumn{4}{|l|}{ Ara h 2/6 } \\
\hline Positive & 19/176 (10.8) & 56/144 (38.9) & $<0.001^{*}$ \\
\hline IgE level, median (IQR) & $0.00(0.00-0.00)$ & $0.00(0.00-6.89)$ & $<0.001^{*}$ \\
\hline \multicolumn{4}{|l|}{ Ara h 3/3.02 } \\
\hline Positive & 10/176 (5.7) & 43/144 (29.9) & $<0.001^{*}$ \\
\hline IgE level, median (IQR) & $0.00(0.00-0.00)$ & $0.00(0.00-0.49)$ & 0.001 \\
\hline \multicolumn{4}{|l|}{ Ara h 8} \\
\hline Positive & 112/176 (63.6) & 67/144 (46.5) & 0.003 \\
\hline IgE level, median (IQR) & $0.44(0.00-1.21)$ & $0.12(0.00-0.82)$ & 0.096 \\
\hline
\end{tabular}

All measurements are in $n / N(\%)$ unless otherwise specified. P-values indicate difference between patients with mild-to-moderate and patients with severe allergic symptoms to peanut. Bold indicates $p<0.05$. 'Differences remained significant after Bonferroni correction. ${ }^{\ddagger}$ Reported symptoms + matching sensitization by SPT or ImmunoCAP. ${ }^{\S}$ Not all patients had complete testing for peanut sensitization. "*Allergen components measured by microarray in 322 patients. IQR, interquartile range; SPT, skin prick test.

\section{Demographic and Clinical Characteristics Associated With Severity of Probable PA}

Frequencies of demographic and clinical background characteristics of patients with mild-to-moderate and those with severe probable PA are presented in Table 1 and Figure 1.
Subjects with the severe phenotype were younger than those with the mild-to-moderate phenotype, and manifestation of probable PA more often occurred before the age of 14 years. Subjects with the severe phenotype were more likely to have symptoms elicited by skin contact with peanut, $\mathrm{AD}, \mathrm{HDM}$ allergy, latex allergy or sensitization to cats and/or dogs, but less likely to be allergic to birch pollen.

\section{Measures of IgE Sensitization Associated With Severity of Probable PA}

Of subjects with probable PA, 320/387 (83\%) had a positive SPT and $284 / 376(76 \%)$ had a positive ImmunoCAP test to peanut extract (Table 1), and 240/370 (65\%) tested positive to both tests. The allergen/histamine wheal ratios and levels of IgE to peanut extract were significantly higher in patients with severe symptoms than in patients with mild-to-moderate symptoms (Table 1 and Figure 1).

Microarray was performed in 322 of 391 (82\%) subjects with probable PA, and 230/322 (71\%) were sensitized to at least one peanut component. All 27 component-sensitized subjects who were not sensitized to peanut extract in SPT or ImmunoCAP, were sensitized to Ara h 8 (Supplementary Table 2). Overall, sensitization to Ara h 8 was most common, and associated with mild-to-moderate probable PA (although not significantly after Bonferroni correction). Sensitization to Ara h 1 , Ara h 2/6 or Ara h 3 was associated with severe probable PA, and IgE levels to these components were significantly higher in those with severe symptoms (Table 1 and Figure 1). Of the 179 subjects with $\operatorname{IgE}$ to Ara h 8 (Table 1), 48 (27\%) also tested positive to Ara h 1, Ara h 2/6 or Ara h 3. Co-sensitization to storage proteins in those sensitized to Ara $\mathrm{h} 8$ was associated with a more severe phenotype $(p=0.009)$.

Regarding foods other than peanut, IgE levels to extract from other legumes, soybean and lentil, were higher in subjects with severe probable PA than in those with mild-to-moderate probable PA (Supplementary Table 3). At a molecular level, subjects with severe probable PA were significantly more often sensitized to soybean Gly $\mathrm{m} 5$ (7S globulin) and Gly $\mathrm{m} 6$ (11S globulin), hazelnut Cor a 11 (7S globulin), walnut Jug r 2 (7S globulin), and sesame Ses i 1 (2S albumin) (Supplementary Table 4). IgE levels to peach, apple and celery extract were higher in subjects with mild-to-moderate probable PA than in subjects with severe probable PA. The mild-to-moderately peanut allergic subjects were more often sensitized to PR 10 proteins Gly m 4 (soybean), Cor a 1 (hazelnut), and Mal d 1 (apple).

\section{Discriminating Between Mild-To-Moderate and Severe Probable PA}

The AUCs of single tests (SPT peanut extract, ImmunoCAP peanut extract, microarray peanut components) for discriminating between patients with mild-to-moderate and severe probable PA ranged from 0.54 to 0.64 (Supplementary Table 5). The accuracy of SPT wheal ratio and of peanut extract and component IgE levels at specific cutpoints, are shown in Supplementary Table 6. The most discriminative model combining microarray results comprised 
A

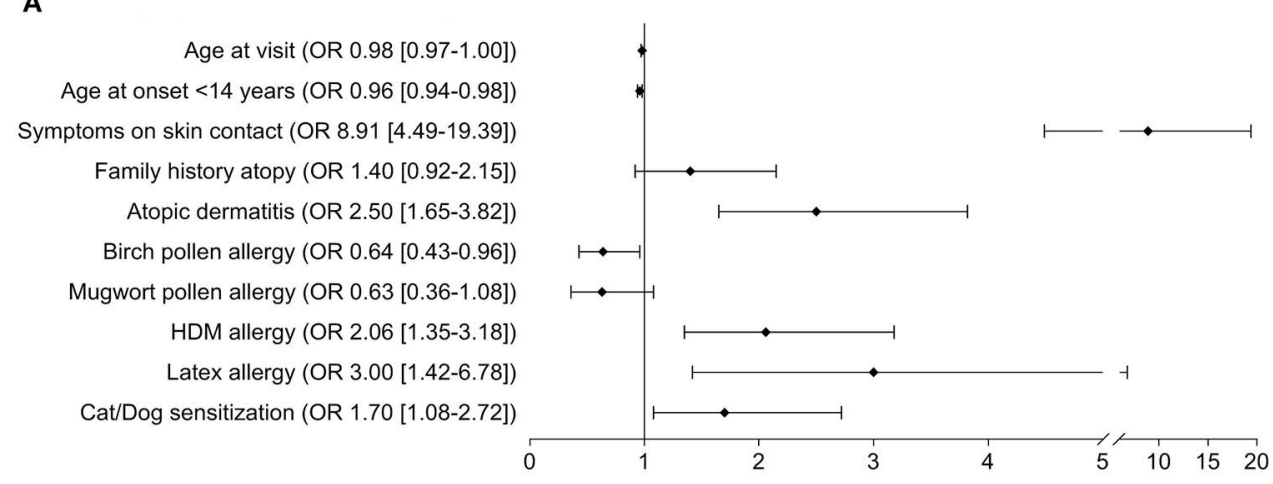

B

SPT peanut extract (OR 2.07 [1.55-2.83])

ImmunoCAP peanut extract (OR 1.02 [1.01-1.03])

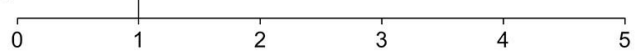

C

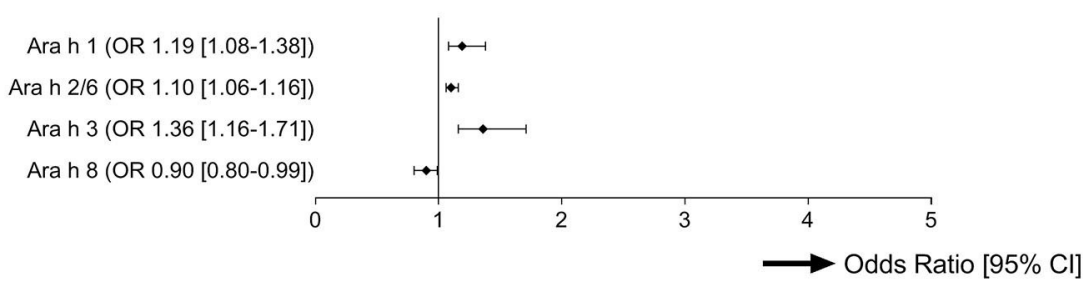

FIGURE 1 | Univariable Odds Ratios for prediction of severity of probable PA $(p<0.2)$. This forest plot shows the ORs and their respective confidence intervals from univariable analyses of all predictors for severity of probable peanut allergy with $p<0.2$ (Table 1). All variables under (B) and (C), and in (A) "age at visit" were entered as continuous variables. All other variables were dichotomous. (A) Demographics and clinical background. (B) Sensitization to peanut extract. (C) Sensitization to peanut components.

IgE levels to Ara h $2 / 6$ and Ara h 8, with an AUC of 0.65 (95\% CI 0.63-0.66). The AUCs of our three models taking demographic and clinical factors as starting point, and combining those with markers for peanut extract and component sensitization, were significantly larger than the AUCs of the single peanut sensitization tests $\left(\mathrm{P}_{\text {De Long's test }}<0.001\right)$ (Table 2 and Supplementary Table 5).

In the first model, female sex, age at onset of PA $<$ 14 years, symptoms elicited by skin contact with peanut, family atopy, $\mathrm{AD}$, birch pollen allergy, HDM allergy, and latex allergy, were selected by Lasso regression. All determinants, except for birch pollen allergy, were associated with severe probable PA. This combination of clinical and demographic factors resulted in an AUC of 0.74 (95\% CI 0.72-0.75). Lasso regression selected SPT wheal size ratio and ImmunoCAP IgE level to peanut extract (both associated with severe PA) as additionally contributing variables in model II, and IgE to Ara h 1 and Ara h 2/6 (severe) and Ara h 8 (mild-tomoderate) in model III, although AUC showed only a limited increase (Table 2). After model III, no IgE levels to foods and food components other than peanut were additionally selected to help discriminate between mild-to-moderate and severe PA.

\section{Discriminating Between Mild-To-Moderate and Severe Symptoms to Peanut in Subjects Who Underwent DBPCFC, or Experienced Severe Life-Threatening Anaphylaxis}

Overall, 52/393 subjects with probable PA agreed to undergo DBPCFC, of which 4 were excluded from analyses because of incomplete data. A total of 91 subjects were included in the subgroup analysis: 47 subjects with no or mild-tomoderate symptoms during DBPCFC (18 subjects with no symptoms, 22 with mild-to-moderate symptoms, 7 placebo-reactors), and 44 subjects with severe symptoms during DBPCFC $(N=1)$ or a convincing history of severe life-threatening anaphylaxis, leading to exclusion from DBPCFC $(N=43)$. Details on demographics, clinical variables, SPT and IgE results are available from Supplementary Table 7. 
TABLE 2 | Prediction models for severity of probable PA.

\begin{tabular}{|c|c|c|c|c|c|c|}
\hline & \multicolumn{2}{|c|}{$\begin{array}{c}\text { Model l: } \\
\text { Demographics } \\
\text { \& clinical background }\end{array}$} & \multicolumn{2}{|c|}{$\begin{array}{c}\text { Model II: } \\
\text { Model I } \\
\text { + sensitization to } \\
\text { peanut extract }\end{array}$} & \multicolumn{2}{|c|}{$\begin{array}{c}\text { Model III: } \\
\text { Model II } \\
+ \text { sensitization to } \\
\text { peanut components }\end{array}$} \\
\hline & OR & $95 \%-\mathrm{Cl}$ & OR & $95 \%-\mathrm{Cl}$ & OR & $95 \%-\mathrm{Cl}$ \\
\hline Age at onset $<14$ years & 1.34 & $0.84-2.13$ & 1.16 & $0.77-1.77$ & 1.15 & $0.77-1.70$ \\
\hline Atopic dermatitis & 1.51 & $0.93-2.44$ & 1.43 & $0.90-2.27$ & 1.46 & $0.91-2.35$ \\
\hline Symptoms skin contact & 5.71 & $2.98-10.93$ & 4.78 & $2.47-9.25$ & 4.57 & 2.33-8.89 \\
\hline Birch pollen allergy & 0.61 & $0.37-1.01$ & 0.63 & $0.38-1.04$ & 0.57 & $0.44-1.15$ \\
\hline HDM allergy & 1.58 & $0.98-2.56$ & 1.47 & $0.91-2.36$ & 1.43 & $0.91-2.25$ \\
\hline Latex allergy & 1.71 & $0.73-4.00$ & 1.73 & $0.78-3.86$ & 1.67 & $0.74-1.58$ \\
\hline IgE level Ara h 8 & & & & & 0.95 & $0.87-1.03$ \\
\hline Intercept & \multicolumn{2}{|l|}{-1.25} & \multicolumn{2}{|l|}{-1.40} & \multicolumn{2}{|l|}{-1.36} \\
\hline AUC (95\%-CI) & \multicolumn{2}{|c|}{$0.74(0.72-0.75)$} & \multicolumn{2}{|c|}{$0.74(0.73-0.76)$} & \multicolumn{2}{|c|}{$0.75(0.74-0.77)$} \\
\hline
\end{tabular}

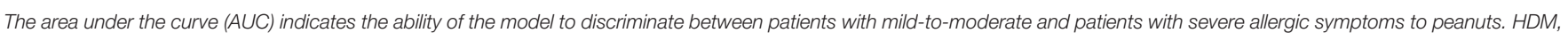
house dust mite; SPT, skin prick test.

TABLE 3 | Prediction models for severity of PA according to DBPCFC or history of anaphylaxis.

\begin{tabular}{|c|c|c|c|c|c|c|}
\hline & \multicolumn{2}{|c|}{$\begin{array}{c}\text { Model I: } \\
\text { Demographics } \\
\text { \& clinical background }\end{array}$} & \multicolumn{2}{|c|}{$\begin{array}{c}\text { Model II: } \\
\text { Model I } \\
+ \text { sensitization to } \\
\text { peanut extract }\end{array}$} & \multicolumn{2}{|c|}{$\begin{array}{c}\text { Model III: } \\
\text { Model II } \\
\text { + sensitization to } \\
\text { peanut components }\end{array}$} \\
\hline & OR & $95 \%-\mathrm{Cl}$ & OR & $95 \%-\mathrm{Cl}$ & OR & $95 \%-\mathrm{Cl}$ \\
\hline Age at visit & 0.95 & $0.90-1.01$ & 0.96 & $0.91-1.02$ & 0.96 & $0.90-1.03$ \\
\hline Symptoms skin contact & 9.93 & $2.22-44.39$ & 9.00 & $1.83-44.33$ & 8.69 & $0.97-77.97$ \\
\hline Birch pollen allergy & 0.64 & $0.19-2.14$ & 0.61 & $0.18-2.14$ & 0.57 & $0.12-2.65$ \\
\hline Grass pollen allergy & 0.39 & $0.09-1.63$ & 0.40 & $0.09-1.76$ & 0.43 & $0.08-2.28$ \\
\hline HDM allergy & 3.11 & $0.75-12.84$ & 2.96 & $0.67-12.99$ & 2.85 & $0.64-12.59$ \\
\hline IgE level peanut extract & & & 1.01 & $0.99-1.03$ & & \\
\hline
\end{tabular}

The area under the curve (AUC) indicates the ability of the model to discriminate between patients with mild-to-moderate and patients with severe allergic symptoms to peanuts. HDM, house dust mite; SPT, skin prick test.

Just like for probable PA, symptoms elicited by skin contact with peanut (associated with severe PA), female sex (severe), family atopy (severe), birch pollen allergy (mild-to-moderate) and HDM allergy (severe) were selected as demographic and clinical predictors for PA in the DBPCFC/anaphylaxis subgroup, with additionally lower age at visit (mild-to-moderate) and grass pollen allergy (mild-to-moderate). IgE to peanut extract (severe) was selected in model II, but no longer in model III, where IgE to Ara h 1 (severe) and Ara h 8 (severe) were favored. The AUC of these models ranged from 0.68 to 0.72 for discriminating between mild-to-moderate and severe PA as determined in the DBPCFC/anaphylaxis subgroup, and did not differ significantly from the AUCs of individual extract- and allergen-based tests (Table 3 and Supplementary Table 5). 


\section{DISCUSSION}

The current study provides insight into the clinical profiles of subjects with mild-to-moderate and severe probable PA, and quantifies the relative importance of information obtained during diagnostic work-up of PA for prediction of severity. Sex, age at onset of PA, symptoms elicited by skin contact with peanut, family atopy, AD (ever), birch pollen allergy, HDM allergy, latex allergy, peanut extract SPT wheal ratio, and IgE levels to peanut extract, Ara h 1, 2/6 and 8, were found to be independently associated with severity, of which only birch pollen allergy and IgE to Ara $\mathrm{h} 8$ were associated with a mild-tomoderate phenotype. A model combining these determinants led to optimal discrimination between mild-to-moderate and severe probable PA (cross-validated AUC 0.75), but measures of peanut sensitization contributed only limited predictive value in addition to clinical background determinants alone.

It was intriguing that some of the strongest independent predictors from clinical background associated with severe probable PA were skin-related: having symptoms elicited by skin contact with peanut, AD (ever), or latex allergy (Figure 1). Exposure to food allergens in early life via the skin has been proposed to play an important role in allergic sensitization (31). Loss-of-function mutations in genes encoding the skin component filaggrin are related to a disrupted skin barrier, are often seen in children with $\mathrm{AD}$, and are associated with $\operatorname{IgE}$ sensitization and allergy to foods in general $(32,33)$, and peanut specifically (33-36). Little has been reported on the relationship between $\mathrm{AD}$ and severity of food allergic reactions, but in agreement with our findings, Van der Leek et al. also found that peanut allergic children reporting skin contact reactions to peanut were more likely to experience severe peanut allergic reactions (37). Similarly, our prediction models developed for hazelnut and walnut allergy also contained $\mathrm{AD}$ (hazelnut and walnut), latex allergy (hazelnut), and symptoms elicited by skin contact (walnut) as predictors for severe reactivity $(20,21)$. Altogether, cutaneous sensitivity may be a marker for severe food allergy.

The only independent determinants to be associated with mild-to-moderate probable PA, were birch pollen allergy and sensitization to Ara h 8, a PR-10 protein homologous to major birch pollen allergen Bet $\mathrm{v} 1$. Birch pollen-related FA is one of the most common types of plant source FA in adults in (especially Northern and Central) Europe and generally presents with mild (often isolated oral allergy) symptoms $(1,38)$. The frequent occurrence of this condition is reflected in our study population-41\% of subjects with mild-to-moderate PA had isolated OAS, of which $73 \%$ were sensitized to Ara h 8 , making birch pollen-related PA plausible.

Interestingly, all subjects with probable PA who were not sensitized to peanut extract in SPT or ImmunoCAP, were found to be sensitized to Ara h 8 (Supplementary Table 2). The peanut PR-10 protein is apparently underrepresented in peanut extract. This suggests that subjects with birch pollen related PA are not well-detected with peanut extract, which partly explains why SPT wheal size and IgE level to peanut extract are associated with severe probable PA. Our findings were similar for walnut allergy, where the majority of subjects with negative extract-based tests were sensitized to walnut PR-10 protein Jug r 5 (21). In contrast, sensitization to hazelnut extract, which is spiked with hazelnut PR-10 protein Cor a 1, is more common in subjects with mild-to-moderate hazelnut allergy (20). In the awareness that the association between extract-based testing and severity of PA was limited, these observations still underline the importance of understanding the allergen composition of food extracts for clinical interpretation of extract-based test results.

Our data showed that levels of IgE to peanut storage proteins Ara h 1, 2/6 and 3 (and also to other legumes', tree nuts' and seeds' storage proteins) were significantly higher in subjects with severe probable PA, in accordance with several previous studies in primarily adult populations $(7,16,39,40)$. Of the individual tests for IgE sensitization to peanut extract or components, IgE to Ara $\mathrm{h} 2 / 6$ had the strongest ability to discriminate between mildto-moderate and severe probable PA, but the AUC only reached 0.64 (Supplementary Table 5). This observation indicated that, although IgE levels to Ara h 1, 2/6 and 3 correlated significantly with severity, they could not be used independently to predict severity of probable PA in an individual patient. These findings were in support of those previously reported by Klemans et al. who also found that IgE to Ara h 2 was associated with severity of PA in their adult population, but could not discriminate well between mild and severe PA in individual patients, with comparable AUCs of 0.58 for severity based on patient history and 0.65 for severity based on DBPCFC (7).

In the current study, IgE to peanut extract (in both SPT and ImmunoCAP) and to peanut storage proteins Ara $\mathrm{h} 1$ and Ara $\mathrm{h} 2 / 6$, were found to contribute to an increased risk of severe probable PA in multivariable analyses. However, the negligible increase of the AUC after addition of measures of peanut $\operatorname{IgE}$ sensitization (in model II and III) to information from clinical background (model I), implies that clinical background is most useful for predicting severity of probable PA in an individual patient, and patient history can detect most of the variation explained by differences in IgE levels. To our knowledge, only one previous study, by Petterson et al. assessed prediction of severity of PA using a combination of variables from clinical background and measures of IgE sensitization (only peanut extract), but in a pediatric population and using linear regression (22). They conclude that reaction severity is largely unpredictable, but the differences in methodological approach prevent in-depth comparison to our study results. Some studies suggest that other laboratory predictors than taken into account in our study may also contribute to prediction of severe PA, such as epitope diversity (combined rather than isolated recognition of Ara $\mathrm{h} 1$, 2 and 3) (41, 42), sIgE/sIgG 4 ratios $(15,43)$, or results from the basophil activation test (BAT) $(15,44)$. Especially the BAT has recently been explored independently and as part of multivariable approaches for prediction of PA severity in several studies. The promising results, albeit in primarily pediatric populations, suggest that the BAT may have the potential to truly enhance prediction of PA severity in the coming years $(43,45-48)$.

Other recommendations for improving prediction of severity of PA in future research, building on the findings in the current study, would be to use ImmunoCAP rather than the less sensitive microarray for measurement of component-specific IgE, and to include other potentially relevant peanut components, like 
profilin Ara h 5, 2S albumin Ara h 7 and lipid transfer protein (LTP) Ara h $9(16,19,49,50)$. The latter is a major peanut allergen in Southern Europe and may contribute to higher predictive accuracy in those regions $(16,51)$. In our population, $\sim 16 \%$ of subjects with probable PA were sensitized to peach LTP Pru p 3 (Supplementary Table 4), which is generally considered the primary source of LTP sensitization (52-54). A previous EuroPrevall study revealed that $73 \%$ of peanut allergic subjects with Pru p 3 sensitization were sensitized to Ara h 9 (16), which suggests that up to $12 \%$ of the subjects with probable PA in our population may have Ara h 9 sensitization. That said, it remains unclear whether knowledge of Ara h 9 sensitization would contribute to prediction of PA severity, as LTP sensitization has been linked to both mild and severe food allergy phenotypes (52), and was not associated with systemic reactions to peanut by Ballmer-Weber et al. (16). In accordance, we also found that sensitization to Pru p 3 was not significantly associated with mild-to-moderate or severe PA in our population (Supplementary Table 4), nor did IgE levels to Pru p 3 improve prediction of PA severity in the multivariable model. The results from the current studies are, for the largest part, based on subjects from birch-endemic areas. It is important to realize that we made the conscious decision to include subjects with likely birch-pollen related PA in our population, even though pollen-related food allergy is considered a separate clinical entity by some. Exclusion of these patients would make the clinical relevance of our findings much more limited for the average presenting outpatient population in most countries in this study. In future research, further specification of the study population to only include subjects from regions with similar pollen exposure, or only children or adults, could further refine prediction and clinical applicability of findings.

One might consider the main limitation of our study that the primary outcome measure was based on self-reported symptoms rather than symptoms during challenge testing. For this reason, we made sure only subjects with IgE sensitization to peanut extract or components were included, and additionally explored the results of our analyses in the subgroup of subjects who underwent challenge testing or were excluded from challenge testing because of a history of severe anaphylaxis. We found it reassuring that there was considerable overlap in independent predictors. It was surprising that Ara h 8 tended to be associated with a more severe phenotype of PA in the DBPCFC/anaphylaxis group, for which we have no clear explanation other than that the subgroup likely does not accurately represent an unselected population of subjects with PA, as subjects with no reaction or reaction to placebo were included in the sub-analysis and the classification of severity in subjects with life-threatening anaphylaxis was based on self-reported symptoms. It is therefore important to realize that the sub-analysis was merely explorative. We also point out that reaction severity based on self-reported symptoms may better reflect real life than reaction severity estimated by challenge, because of exclusion and stopping criteria, and the disinclination of patients who experience severe reactions to undergo or complete a burdensome challenge. As a result of the latter, dietary avoidance advice and medical prescriptions in daily practice are often decided based on clinical history and measurements of IgE sensitization, making models predicting severity of probable PA particularly interesting. We used penalized regression to prevent overfitting of our models to the population in which they were developed, but as with all prediction models, the models should still be validated in an external population.

To our knowledge, this is the first study to evaluate the individual and combined contribution of clinical background, extract-based tests, and CRD, for prediction of PA severity in a primarily adult population. The penalized regression method increases the generalizability of results, and the standardized approach facilitates comparison to similar models designed for tree nuts. Although not superimposable, clinical profiles for hazelnut and walnut displayed clear similarities. However, it was interesting to observe that measurements of IgE sensitization only contributed minimally to prediction of severity of probable $\mathrm{PA}$, in contrast to the models for severity of hazelnut or walnut allergy. Clinical background determinants were clearly most valuable for predicting severity of probable PA in an individual patient. It will be interesting to validate and further expand these models in other populations to increase predictive accuracy, and to develop models according to the same approach in other food groups for comparative purposes.

\section{DATA AVAILABILITY STATEMENT}

The datasets presented in this article are not readily available because the EuroPrevall datasets are only available by request to the original coordinator of the EuroPrevall Project, E. N. Clare Mills. A Data Access Committee decides whether the data will be made accessible. Requests to access the datasets should be directed to clare.mills@manchester.ac.uk.

\section{ETHICS STATEMENT}

The studies involving human participants were reviewed and approved by the local ethical committee of each of the 12 participating centers. Written informed consent to participate in this study was provided by the participants' legal guardian/next of kin.

\section{AUTHOR CONTRIBUTIONS}

EM and RR: coordination of EuroPrevall project. MF-R: coordinator of EuroPrevall outpatient clinic survey design of survey and protocol writing. MF-R and BB-W: training of the clinical partners and anaphylaxis board. BB-W: coordination of the food challenges in EuroPrevall. MF-R, BB-W, AK, RA, SB, FB, MC, RD, PF, DG, MJ-C, MK, TK, TP, AP, IR, SS, T-ML, and EV: recruitment and clinical investigation of the patients and food challenge performance. KH-S: coordination of allergen bank. JL: generation of reagents and experimental IgE assays. SV, SAV, and LJ: immunoCAP IgE testing (extract based). MF-R, LB, and CF-P: database design, data collection, and data cleaning. LB, CF-P, NP, and MF-R: monitoring data collection and data cleaning. MD and SL: statistical data analyses and writing of the manuscript. 
$A Z$ and PW: supervision of the statistical data analyses. MF-R, BB-W, AK, RA, LB, SB, FB, MC, RD, CF-P, PF, DG, KH-S, MJ-C, LJ, MK, TK, JL, NGP, TP, NP, AP, IR, SS, AS, EV, SAV, SV, PW, EM, T-ML, AZ, and RR: critical review of the manuscript. All authors contributed to the article and approved the submitted version.

\section{FUNDING}

This work was funded by the European Commission under the 6th Framework Programme through EuroPrevall (FP6- FOODCT-2005-514000) and the 7th Framework Programme through iFAAM (Grant agreement no. 312147), respectively.

\section{REFERENCES}

1. Turner PJ, Baumert JL, Beyer K, Boyle RJ, Chan CH, Clark AT, et al. Can we identify patients at risk of life-threatening allergic reactions to food? Allergy. (2016) 71:1241-55. doi: 10.1111/all.12924

2. Greenhawt M, Shaker M, Wang J, Oppenheimer JJ, Sicherer S, Keet C, et al. Peanut allergy diagnosis: a 2020 practice parameter update, systematic review, and gRADE analysis. J Allergy Clin Immunol. (2020) 146:130234. doi: 10.1016/j.jaci.2020.07.031

3. Wainstein BK, Studdert J, Ziegler M, Ziegler JB. Prediction of anaphylaxis during peanut food challenge: usefulness of the peanut skin prick test (SPT) and specific IgE level. Pediatr Allergy Immunol. (2010) 21:60311. doi: 10.1111/j.1399-3038.2010.01063.x

4. Summers CW, Pumphrey RS, Woods CN, McDowell G, Pemberton PW, Arkwright PD. Factors predicting anaphylaxis to peanuts and tree nuts in patients referred to a specialist center. J Allergy Clin Immunol. (2008) 121:6328.e2. doi: 10.1016/j.jaci.2007.12.003

5. Ta V, Weldon B, Yu G, Humblet O, Neale-May S, Nadeau K. Use of specific $\mathrm{IgE}$ and skin prick test to determine clinical reaction severity. $\mathrm{Br} \mathrm{J} \mathrm{Med} \mathrm{Med}$ Res. (2011) 1:410-29. doi: 10.9734/BJMMR/2011/711

6. Klemans RJ, Otte D, Knol M, Knol EF, Meijer Y, Gmelig-Meyling FH, et al. The diagnostic value of specific igE to ara $\mathrm{h} 2$ to predict peanut allergy in children is comparable to a validated and updated diagnostic prediction model. J Allergy Clin Immunol. (2013) 131:157-63. doi: 10.1016/j.jaci.2012.8.010

7. Klemans RJ, Broekman HC, Knol EF, Bruijnzeel-Koomen CA, Otten HG, Pasmans SG, et al. Ara h 2 is the best predictor for peanut allergy in adults. $J$ Allergy Clin Immunol Pract. (2013) 1:632-8.e1. doi: 10.1016/j.jaip.2013.07.014

8. Nicolaou N, Poorafshar M, Murray C, Simpson A, Winell H, Kerry G, et al. Allergy or tolerance in children sensitized to peanut: prevalence and differentiation using component-resolved diagnostics. J Allergy Clin Immunol. (2010) 125:191-7.e1-13. doi: 10.1016/j.jaci.2009.10.008

9. Eller E, Bindslev-Jensen C. Clinical value of component-resolved diagnostics in peanut-allergic patients. Allergy. (2013) 68:190-4. doi: 10.1111/all.12075

10. Beyer K, Grabenhenrich L, Hartl M, Beder A, Kalb B, Ziegert M, et al. Predictive values of component-specific IgE for the outcome of peanut and hazelnut food challenges in children. Allergy. (2015) 70:908. doi: $10.1111 /$ all.12530

11. Martinet J, Couderc L, Renosi F, Bobee V, Marguet C, Boyer O. Diagnostic value of antigen-Specific immunoglobulin e Immunoassays against Ara $\mathrm{h}$ 2 and Ara h 8 peanut components in child food allergy. Int Arch Allergy Immunol. (2016) 169:216-22. doi: 10.1159/000446181

12. van Veen LN, Heron M, Batstra M, van Haard PMM, de Groot H. The diagnostic value of component-resolved diagnostics in peanut allergy in children attending a regional paediatric allergology clinic. BMC Pediatr. (2016) 16:74. doi: 10.1186/s12887-016-0609-7

13. van Erp FC, Knol EF, Pontoppidan B, Meijer Y, van der Ent CK, Knulst AC. The IgE and basophil responses to Ara h 2 and Ara h 6 are good predictors of peanut allergy in children. J Allergy Clin Immunol. (2017) 139:358-60.e8. doi: 10.1016/j.jaci.2016.06.041

\section{ACKNOWLEDGMENTS}

We would like to thank all the patients for their participation in the study. We would like to thank ALK Abello (Madrid, Spain) for their generous gift of SPT reagents. We acknowledge the support by the 6th and 7th Framework Programmes of the EU, for EuroPrevall (FP6-FOOD-CT-2005-514000) and iFAAM (Grant agreement no. 312147), respectively.

\section{SUPPLEMENTARY MATERIAL}

The Supplementary Material for this article can be found online at: https://www.frontiersin.org/articles/10.3389/falgy. 2021.670789/full\#supplementary-material

14. Datema MR, Eller E, Zwinderman AH, Poulsen LK, Versteeg SA, van Ree R, et al. Ratios of specific IgG4 over IgE antibodies do not improve prediction of peanut allergy nor of its severity compared to specific igE alone. Clin Exp Allergy. (2019) 49:216-26. doi: 10.1111/cea.13286

15. Song Y, Wang J, Leung N, Wang LX, Lisann L, Sicherer SH, et al. Correlations between basophil activation, allergen-specific IgE with outcome and severity of oral food challenges. Ann Allergy Asthma Immunol. (2015) 114:31926. doi: 10.1016/j.anai.2015.01.006

16. Ballmer-Weber BK, Lidholm J, Fernandez-Rivas M, Seneviratne S, Hanschmann KM, Vogel L, et al. IgE recognition patterns in peanut allergy are age dependent: perspectives of the euroPrevall study. Allergy. (2015) 70:391-407. doi: 10.1111/all.12574

17. Blumchen K, Beder A, Beschorner J, Ahrens F, Gruebl A, Hamelmann E, et al. Modified oral food challenge used with sensitization biomarkers provides more real-life clinical thresholds for peanut allergy. J Allergy Clin Immunol. (2014) 134:390-8. doi: 10.1016/j.jaci.2014. 03.035

18. Deschildre A, Elegbede CF, Just J, Bruyere O, Van der Brempt X, Papadopoulos A, et al. Peanut-allergic patients in the MIRABEL survey: characteristics, allergists' dietary advice and lessons from real life. Clin Exp Allergy. (2016) 46:610-20. doi: 10.1111/cea.12681

19. Muraro A, Werfel T, Hoffmann-Sommergruber K, Roberts G, Beyer K, Bindslev-Jensen $\mathrm{C}$, et al. EAACI food allergy and anaphylaxis guidelines: diagnosis and management of food allergy. Allergy. (2014) 69:100825. doi: 10.1111/all.12429

20. Datema MR, van Ree R, Asero R, Barreales L, Belohlavkova S, de Blay F, et al. Component-resolved diagnosis and beyond: multivariable regression models to predict severity of hazelnut allergy. Allergy. (2018) 73:54959. doi: 10.1111/all.13328

21. Lyons SA, Datema MR, Le TM, Asero R, Barreales L, Belohlavkova S, et al. Walnut allergy across Europe: distribution of allergen sensitization patterns and prediction of severity. J Allergy Clin Immunol Pract. (2020) 9:225-235.e10. doi: 10.1016/j.jaip.2020.08.051

22. Pettersson ME, Koppelman GH, Flokstra-de Blok BMJ, Kollen BJ, Dubois AEJ. Prediction of the severity of allergic reactions to foods. Allergy. (2018) 73:1532-40. doi: 10.1111/all.13423

23. DunnGalvin A, Daly D, Cullinane C, Stenke E, Keeton D, Erlewyn-Lajeunesse $\mathrm{M}$, et al. Highly accurate prediction of food challenge outcome using routinely available clinical data. J Allergy Clin Immunol. (2011) 127:633-9.e13. doi: 10.1016/j.jaci.2010.12.004

24. Fernandez-Rivas M, Barreales L, Mackie AR, Fritsche P, Vazquez-Cortes S, Jedrzejczak-Czechowicz M, et al. The euroPrevall outpatient clinic study on food allergy: background and methodology. Allergy. (2015) 70:57684. doi: 10.1111 /all.12585

25. Hoffmann-Sommergruber K, Mills EN, Vieths S. Coordinated and standardized production, purification and characterization of natural and recombinant food allergens to establish a food allergen library. Mol Nutr Food Res. (2008) 52(Suppl 2):S159-65. doi: 10.1002/mnfr. 200800466 
26. Cochrane SA, Salt LJ, Wantling E, Rogers A, Coutts J, Ballmer-Weber BK, et al. Development of a standardized low-dose double-blind placebocontrolled challenge vehicle for the euroPrevall project. Allergy. (2012) 67:107-13. doi: 10.1111/j.1398-9995.2011.02715.x

27. Muraro A, Fernandez-Rivas M, Beyer K, Cardona V, Clark A, Eller E, et al. The urgent need for a harmonized severity scoring system for acute allergic reactions. Allergy. (2018) 73:1792-800. doi: 10.1111/all.13408

28. Sampson HA. Anaphylaxis and emergency treatment. Pediatrics. (2003) 111:1601-8.

29. Tibshirani R. Regression shrinkage and selection via the lasso. J Roy Stat Soc B. (1996) 58:267-88. doi: 10.1111/j.2517-6161.1996.tb02080.x

30. DeLong ER, DeLong DM, Clarke-Pearson DL. Comparing the areas under two or more correlated receiver operating characteristic curves: a nonparametric approach. Biometrics. (1988) 44:837-45. doi: 10.2307/2531595

31. Lack G. Update on risk factors for food allergy. J Allergy Clin Immunol. (2012) 129:1187-97. doi: 10.1016/j.jaci.2012.02.036

32. Tsakok T, Marrs T, Mohsin M, Baron S, du Toit G, Till S, et al. Does atopic dermatitis cause food allergy? A systematic review. J Allergy Clin Immunol. (2016) 137:1071-8. doi: 10.1016/j.jaci.2015.10.049

33. Venkataraman D, Soto-Ramirez N, Kurukulaaratchy RJ, Holloway JW, Karmaus W, Ewart SL, et al. Filaggrin loss-of-function mutations are associated with food allergy in childhood and adolescence. J Allergy Clin Immunol. (2014) 134:876-82.e4. doi: 10.1016/j.jaci.2014.07.033

34. Hill DJ, Sporik R, Thorburn J, Hosking CS. The association of atopic dermatitis in infancy with immunoglobulin e food sensitization. J Pediatr. (2000) 137:475-9. doi: 10.1067/mpd.2000.108207

35. Sampson HA, Broadbent KR, Bernhisel-Broadbent J. Spontaneous release of histamine from basophils and histamine-releasing factor in patients with atopic dermatitis and food hypersensitivity. N Engl J Med. (1989) 321:22832. doi: 10.1056/NEJM198907273210405

36. Brown SJ, Asai Y, Cordell HJ, Campbell LE, Zhao Y, Liao H, et al. Loss-of-function variants in the filaggrin gene are a significant risk factor for peanut allergy. J Allergy Clin Immunol. (2011) 127:6617. doi: 10.1016/j.jaci.2011.01.031

37. Vander Leek TK, Liu AH, Stefanski K, Blacker B, Bock SA. The natural history of peanut allergy in young children and its association with serum peanut-specific IgE. J Pediatr. (2000) 137:749-55. doi: 10.1067/mpd.2000. 109376

38. Werfel T, Asero R, Ballmer-Weber BK, Beyer K, Enrique E, Knulst AC, et al. Position paper of the EAACI: food allergy due to immunological cross-reactions with common inhalant allergens. Allergy. (2015) 70:107990. doi: 10.1111/all.12666

39. Peeters KA, Koppelman SJ, van Hoffen E, van der Tas CW, den Hartog Jager $\mathrm{CF}$, Penninks AH, et al. Does skin prick test reactivity to purified allergens correlate with clinical severity of peanut allergy? Clin Exp Allergy. (2007) 37:108-15. doi: 10.1111/j.1365-2222.2006.02628.x

40. Moverare R, Ahlstedt S, Bengtsson U, Borres MP, van Hage M, Poorafshar $\mathrm{M}$, et al. Evaluation of $\mathrm{IgE}$ antibodies to recombinant peanut allergens in patients with reported reactions to peanut. Int Arch Allergy Immunol. (2011) 156:282-90. doi: 10.1159/000323891

41. Flinterman AE, Knol EF, Lencer DA, Bardina L, den Hartog Jager CF, Lin J, et al. Peanut epitopes for IgE and IgG4 in peanut-sensitized children in relation to severity of peanut allergy. J Allergy Clin Immunol. (2008) 121:737-43 e10. doi: 10.1016/j.jaci.2007.11.039

42. Shreffler WG, Beyer K, Chu TH, Burks AW, Sampson HA. Microarray immunoassay: association of clinical history, in vitro $\operatorname{IgE}$ function, and heterogeneity of allergenic peanut epitopes. J Allergy Clin Immunol. (2004) 113:776-82. doi: 10.1016/j.jaci.2003.12.588

43. Santos AF, Du Toit G, O'Rourke C, Becares N, Couto-Francisco N, Radulovic S, et al. Biomarkers of severity and threshold of allergic reactions during oral peanut challenges. J Allergy Clin Immunol. (2020) 146:34455. doi: 10.1016/j.jaci.2020.03.035

44. Santos AF, Du Toit G, Douiri A, Radulovic S, Stephens A, Turcanu V, et al. Distinct parameters of the basophil activation test reflect the severity and threshold of allergic reactions to peanut. J Allergy Clin Immunol. (2015) 135:179-86. doi: 10.1016/j.jaci.2014.09.001
45. Chinthrajah RS, Purington N, Andorf S, Rosa JS, Mukai K, Hamilton $\mathrm{R}$, et al. Development of a tool predicting severity of allergic reaction during peanut challenge. Ann Allergy Asthma Immunol. (2018) 121:69-76 e2. doi: 10.1016/j.anai.2018.04.020

46. Cottel N, Saf S, Bourgoin-Heck M, Lambert N, Amat F, Poncet P, et al. Two different composite markers predict severity and threshold dose in peanut allergy. J Allergy Clin Immunol Pract. (2020) 9:27582.e1. doi: 10.1016/j.jaip.2020.09.043

47. Buyuktiryaki B, Santos AF. Food allergy severity predictions based on cellular in vitro tests. Expert Rev Mol Diagn. (2020) 20:679-92. doi: 10.1080/14737159.2020.1782192

48. Santos AF. Food allergy severity prediction: quite a way to go yet? Expert Rev Clin Immunol. (2020) 16:543-6. doi: 10.1080/1744666X.2020.1770083

49. Palladino C, Breiteneder H. Peanut allergens. Mol Immunol. (2018) 100:58 70. doi: 10.1016/j.molimm.2018.04.005

50. Blankestijn MA, Otten HG, Suer W, Weimann A, Knol EF, Knulst AC. Specific IgE to peanut $2 S$ albumin Ara $\mathrm{h} 7$ has a discriminative ability comparable to Ara h 2 and 6. Clin Exp Allergy. (2018) 48:60-5. doi: 10.1111/cea.13030

51. Vereda A, van Hage M, Ahlstedt S, Ibanez MD, Cuesta-Herranz J, van Odijk J, et al. Peanut allergy: clinical and immunologic differences among patients from 3 different geographic regions. J Allergy Clin Immunol. (2011) 127:603-7. doi: 10.1016/j.jaci.2010.09.010

52. Asero R. In patients with ITP syndrome food-specific IgE show a predictable hierarchical order. Eur Ann Allergy Clin Immunol. (2014) 46:142-6.

53. Egger M, Hauser M, Mari A, Ferreira F, Gadermaier G. The role of lipid transfer proteins in allergic diseases. Curr Allergy Asthma Rep. (2010) 10:32635. doi: 10.1007/s11882-010-0128-9

54. Lauer I, Miguel-Moncin MS, Abel T, Foetisch K, Hartz C, Fortunato D, et al. Identification of a plane pollen lipid transfer protein (Pla a 3) and its immunological relation to the peach lipid-transfer protein, pru p 3. Clin Exp Allergy. (2007) 37:261-9.doi: 10.1111/j.1365-2222.2007.02653.x

Conflict of Interest: Outside of submitted work: MF-R reported grants and personal fees from Aimmune Therapeutics and Diater, personal fees from DBV, Allergy Therapeutics, GSK, HAL Allergy, Novartis, ThermoFisher Scientific, and SPRIM. BB-W reported personal fees from ThermoFisher Scientific. FB reported personal fees from Aimmune; grants from Stallergènes Greer, Chiesi, Mundipharma, Novartis, and Regeneron; and board membership for DVB, Stallergènes Greer, Novartis, ALK, Mundipharma, Boehringer, AstraZeneca, Medapharma, and Boston Scientific. JL was an employee of ThermoFisher Scientific. NP reported personal fees from Novartis, Nutricia, HAL Allergy BV, Menarine/Faes Farma, Sanofi, Mylan/Meda, Biomay, AstraZeneca, GSK, MSD, ASIT Biotech, Boehringer Ingelheim; and grants from Gerolymatos International SA, and Capricare. SV reported personal fees from Ärzteverband Deutscher Allergologen, Swiss Society for Allergy and Immunology, Schattauer Allergologie Handbuch, Elsevier Nahrungsmittelallergien und Intoleranzen, Karger Food Allergy: Molecular Basis and Clinical Practice, and Pharmacon. EM reported grants from Reacta Biotech; and was shareholder of Reacta Biotech Ltd. RR reported personal fees from HAL Allergy BV, Citeq BV, Angany Inc., and ThermoFisher Scientific.

The remaining authors declare that the research was conducted in the absence of any commercial or financial relationships that could be construed as a potential conflict of interest.

Copyright (C) 2021 Datema, Lyons, Fernández-Rivas, Ballmer-Weber, Knulst, Asero, Barreales, Belohlavkova, de Blay, Clausen, Dubakiene, Fernández-Perez, Fritsche, Gislason, Hoffmann-Sommergruber, Jedrzejczak-Czechowicz, Jongejan, Kowalski, Kralimarkova, Lidholm, Papadopoulos, Popov, Prado, Purohit, Reig, Seneviratne, Sinaniotis, Vassilopoulou, Versteeg, Vieths, Welsing, Mills, Le, Zwinderman and van Ree. This is an open-access article distributed under the terms of the Creative Commons Attribution License (CC BY). The use, distribution or reproduction in other forums is permitted, provided the original author(s) and the copyright owner(s) are credited and that the original publication in this journal is cited, in accordance with accepted academic practice. No use, distribution or reproduction is permitted which does not comply with these terms. 